ARTICLE

\title{
A microsporidian impairs Plasmodium falciparum transmission in Anopheles arabiensis mosquitoes
}

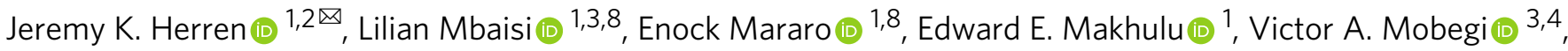 \\ Hellen Butungi (1) 1,5, Maria Vittoria Mancini (1) 2, Joseph W. Oundo (10) 1, Evan T. Teal (1) 1, Silvain Pinaud (1) ${ }^{6}$,

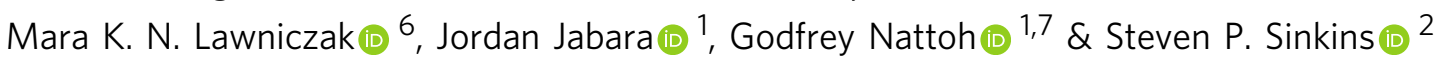

\begin{abstract}
A possible malaria control approach involves the dissemination in mosquitoes of inherited symbiotic microbes to block Plasmodium transmission. However, in the Anopheles gambiae complex, the primary African vectors of malaria, there are limited reports of inherited symbionts that impair transmission. We show that a vertically transmitted microsporidian symbiont (Microsporidia MB) in the An. gambiae complex can impair Plasmodium transmission. Microsporidia $M B$ is present at moderate prevalence in geographically dispersed populations of An. arabiensis in Kenya, localized to the mosquito midgut and ovaries, and is not associated with significant reductions in adult host fecundity or survival. Field-collected Microsporidia MB infected An. arabiensis tested negative for $P$. falciparum gametocytes and, on experimental infection with $P$. falciparum, sporozoites aren't detected in Microsporidia MB infected mosquitoes. As a microbe that impairs Plasmodium transmission that is non-virulent and vertically transmitted, Microsporidia MB could be investigated as a strategy to limit malaria transmission.
\end{abstract}

\footnotetext{
${ }^{1}$ International Centre of Insect Physiology and Ecology (ICIPE), Kasarani, Nairobi, Kenya. ${ }^{2}$ MRC-University of Glasgow Centre for Virus Research, 464 Bearsden Road, Glasgow G61 1QH, UK. ${ }^{3}$ Centre for Biotechnology and Bioinformatics (CEBIB), University of Nairobi, Nairobi, Kenya. ${ }^{4}$ Department of Biochemistry, University of Nairobi, Nairobi, Kenya. ${ }^{5}$ University of the Witwaterstrand, Wits Research Institute for Malaria, Johannesburg, South Africa. ${ }^{6}$ Wellcome Sanger Institute, Wellcome Genome Campus, Hinxton CB10 1SA, UK. 7 Pan African University Institute for Basic Sciences Technology \& Innovation, Nairobi, Kenya. ${ }^{8}$ These authors contributed equally: Lilian Mbaisi, Enock Mararo. ${ }^{凶}$ email: jherren@icipe.org
} 
T he malaria disease burden remains a major impediment to economic development over many regions of sub-Saharan Africa. Large-scale insecticide treated net (ITN) distribution campaigns over the previous 15 years have reduced malaria cases by an estimated $40 \%{ }^{1}$. However, progress has plateaued; between 2014 and 2016 global incidence remained essentially the same ${ }^{1}$. This is a strong indication that current control measures are insufficient and additional novel strategies to control Anopheles mosquito populations or their capacity to transmit Plasmodium parasites are needed if we are to make further inroads in reducing malaria incidence.

The outcome of vector-pathogen interactions can be influenced by symbiotic microbes. Notably, symbionts can prevent disease vectors from transmitting pathogens that are agents of human disease. This can be developed into a novel vector management strategy; symbionts are disseminated into vector populations to limit their capacity to transmit human disease. For example, it has been demonstrated that Wolbachia, which protects mosquitoes from viral pathogens, has great potential for controlling the spread of mosquito-transmitted viral diseases of humans $^{2-7}$. There are reports of Wolbachia from anopheline mosquitoes $^{8,9}$, in some cases with evidence for a Plasmodiumprotective phenotype ${ }^{10,11}$. However, there are also indications that Wolbachia strains in An. gambiae s.l. are either low intensity and have low efficiency of vertical transmission ${ }^{12}$, or are artefacts produced by contamination ${ }^{13}$. Being intracellular and maternally inherited, many microsporidians have a similar lifestyle to $\mathrm{Wol}$ bachia. These obligately intracellular simple eukaryotes, classified within or as a sister group to fungi ${ }^{14}$, are known to be harbored by mosquitoes and could be equally useful for the control of vector-borne diseases.

Microsporidia have lifecycles that include a meront phase during proliferation, and spores with chitinous cell walls involved in host to host transmission through spore ingestion. Species with solely horizontal transmission usually show greater virulence and lower host specificity, but where a combination of horizontal and vertical transovarial transmission occurs, lower virulence is advantageous, and is normally associated with a higher degree of host specificity ${ }^{15}$. Sex ratio distortion toward females has been reported (a manipulation characteristic of transovarially transmitted symbionts), for example in the Dictyocoela microsporidian of Amphipod crustaceans ${ }^{16}$. Various Microsporidia species have been reported in mosquitoes ${ }^{17-26}$, with simple or complex lifecycles ${ }^{19}$ but all of which are pathogens where virulence is primarily associated with larval mortality or reduced adult fecundity and lifespan ${ }^{18-23}$. Here, we characterize an apparently nonpathogenic microsporidian from field populations of An. arabiensis in Kenya. We link Microsporidia infection to the reduction of Plasmodium transmission capacity in this important vector species.

\section{Results}

Microsporidia detected in An. arabiensis field populations. A microsporidian, designated Microsporidia $M B$, was found to occur at a high intensity and with low to moderate prevalence $(0-9 \%)$ in geographically dispersed populations of An. arabiensis in Kenya (Supplementary Fig. 1). Phylogenetic analysis of the $18 \mathrm{~S}$ ribosomal gene revealed that Microsporidia $M B$ is related to Crispospora chironomi ${ }^{27}$, a species recently identified from nonbiting midges. The $18 S$ gene sequence of Microsporidia $M B$ shows 97\% similarity with Crispospora chironomi. Microsporidia MB and Crispospora chironomi are in clade IV that unites Microsporidia of terrestrial origin infecting diverse hosts ${ }^{28}$ (Fig. 1a and Supplementary Fig. 2). The previous reports of microsporidians infecting Anopheles mosquitoes all belong to different clades of
Microsporidia ${ }^{28}$. The morphology of Microsporidia $M B$ closely resembles Crispospora chironomi, exhibiting both polysporoblastic and diplosporoblastic sporogenies, both found in the larval mosquito gut epithelium (Fig. 1b).

Seasonal variation in Microsporidia MB prevalence. In Kenya, there is a bimodal precipitation pattern, with long rains generally occurring from April to June and short rains between November and December. By screening field-collected An. arabiensis mosquitoes we established that Microsporidia $M B$ prevalence fluctuates seasonally, with the greatest proportion of infected $A n$. arabiensis being observed in January and June, 4-6 weeks after peak rainfall in the Mwea collection site (Fig. 2). At another collection site, Ahero, Microsporidia $M B$ prevalence also increased after the rains; however, the timing of this rise was less distinct. Notably, this region has less clearly defined periods of peak rainfall (Fig. 2).

Microsporidia $M B$ is vertically transmitted. Microsporidia $M B$ are maternally transmitted with high efficiency, from 45 to $100 \%$ (Fig. 3a). The intensity of Microsporidia $M B$ in $\mathrm{G}_{1}$ offspring is positively correlated to maternal $G_{o}$ intensity (Fig. 3b). Microsporidia $M B$ were observed in the mosquito ovaries, where the symbiont colonizes and penetrates oocytes (Fig. 3c). We found no evidence that Microsporidia $M B$ manipulates the sex ratio of $A n$. arabiensis (Fig. 3d).

Microsporidia MB impairs Plasmodium transmission. The effects of Microsporidia MB on Plasmodium infection in An. arabiensis was further examined around Mbita point, western Kenya, using $G_{1}$ offspring pools obtained from field-collected $G_{0}$ mosquitoes and direct membrane feeding assays (DMFAs) carried out with Plasmodium falciparum infected donor blood. Since there was a low to moderate prevalence of Microsporidia $M B$ in field populations (Fig. 4, Supplementary Fig. 1), we screened mosquitoes for Microsporidia $M B$ and sorted them to ensure that $\mathrm{G}_{1}$ progeny pools used for DMFAs would have both Microsporidia $M B$ infected and uninfected mosquitoes. Plasmodium was quantified by $\mathrm{qPCR} 10$ days after DMFAs, and a strong negative correlation was apparent between Microsporidia $M B$ and the presence of Plasmodium in whole mosquitoes (Supplementary Fig. 3).

Plasmodium parasites in an ingested bloodmeal undergo a series of developmental changes prior to traversing the peritrophic matrix and midgut epithelium to form a sporogonic oocyst, which releases sporozoites into the hemocoel. From the hemocoel, sporozoites travel to the salivary gland, traverse an epithelium and mix with Anopheles saliva resulting in an infectious mosquito, usually $8-14$ days after the blood meal ${ }^{29}$. To investigate the stage at which Plasmodium development was impaired, we specifically quantified Plasmodium in the An. arabiensis head and thorax and abdominal compartments. The absence of Plasmodium in the head and thorax compartment of Microsporidia $M B$ infected An. arabiensis 10 days after DMFA indicates that Microsporidia $M B$ prevents An. arabiensis salivary glands from being colonized by Plasmodium sporozoites (Fig. 4a, b) and therefore prevents Plasmodium transmission. In addition, Plasmodium was not detected by $\mathrm{qPCR}$ in the abdomens of Microsporidia $M B$ infected An. arabiensis 10 days after DMFA (Fig. 4c, d). The absence of Plasmodium detected by qPCR in the abdomens of Microsporidia MB infected An. arabiensis indicates a strong reduction in the development of Plasmodium oocysts in the mosquito midgut ${ }^{30}$. To determine to what extent the $\mathrm{qPCR}$ assay was able to accurately identify the absence of Plasmodium oocysts we carried out both qPCR and microscopic examination 
a

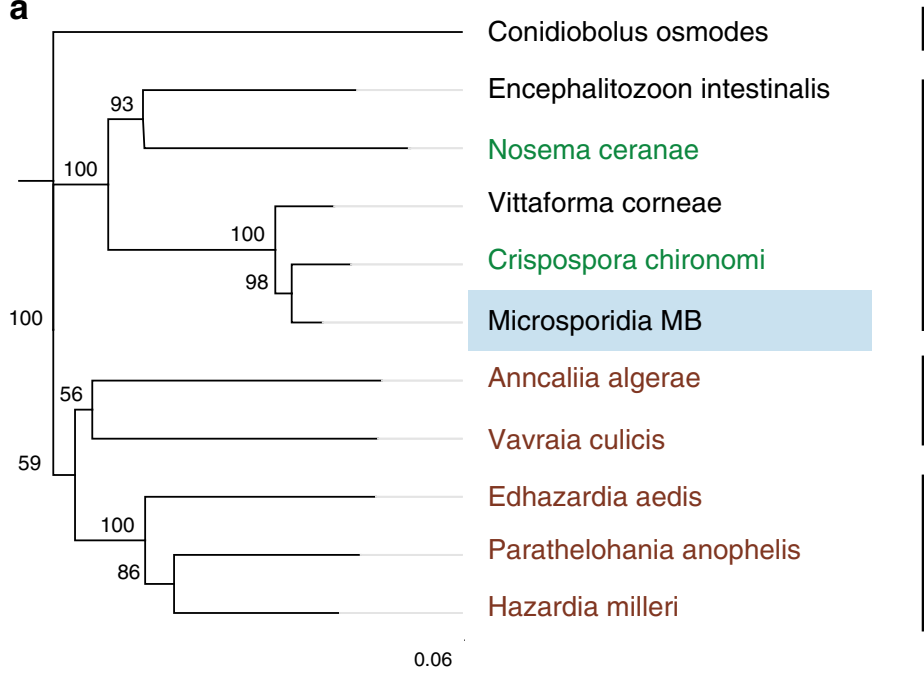

Outgroup

clade IV

clade III

clade I

b
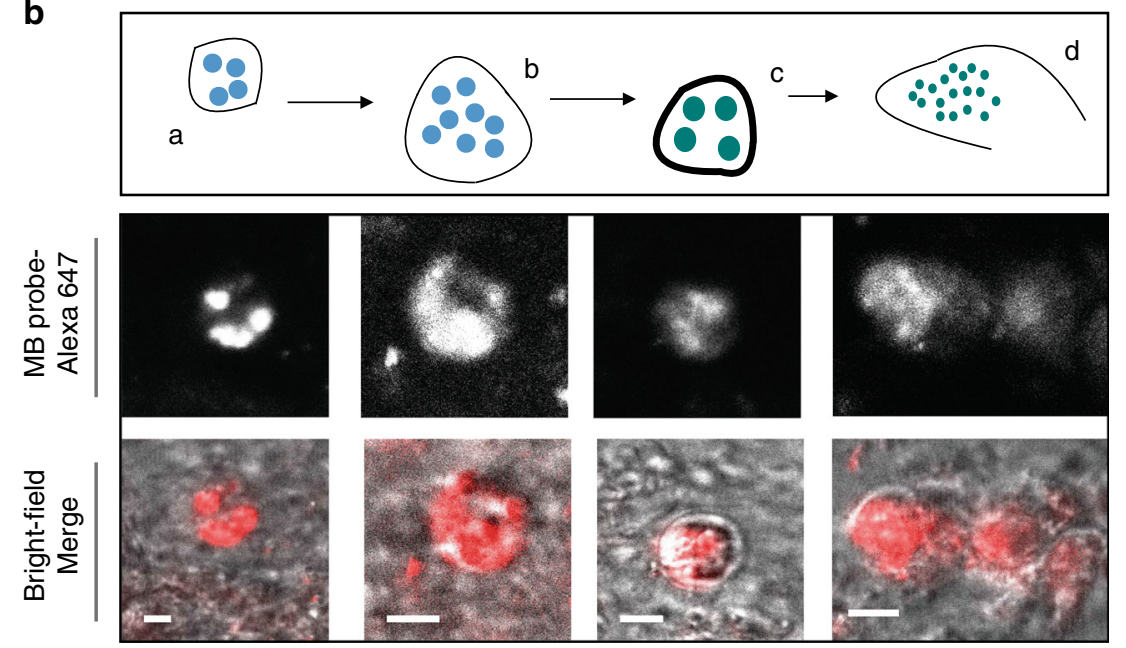

Fig. 1 A microsporidian associated with An. arabiensis populations in Kenya. a 185 rDNA-based phylogeny reveals that Microsporidia MB are in clade IV of the Microsporidia. Labeled in brown are the microsporidian species known from mosquitoes. In green are microsporidians associated with other insect groups. Microsporidia MB $18 \mathrm{~S}$ rDNA gene sequence deposited in GenBank (accession number MT160806[https://www.ncbi.nlm.nih.gov/nuccore/ MT160806]) and in Source Data file. b Fluorescence in situ hybridization (FISH) staining of the diplokaryotic stages of Microsporidia MB merogony (a, b) and spore capsule formation (c, d) in An. arabiensis larval gut epithelial tissues. Red in the merge images is the MB probe-Alexa $647 \mathrm{FISH}$ probe targeting Microsporidia MB. The scale bar is $1.5 \mu \mathrm{m}$. Representative images as observed in four independent experiments.

on the same 75 Anopheles midguts (Supplementary Table 1). Notably, we observed greater sensitivity in the qPCR-based assay, which is in concordance with other PCR-based studies that observed higher sensitivity for Plasmodium DNA detection than other methods ${ }^{31,32}$. The qPCR-based assay detected Plasmodium in 26 midguts, whereas only 21 midguts were found to be positive based on microscopy. We note that 2 midguts were found to have a low number of oocysts by microscopy $(1,3)$ and were not found to have Plasmodium based on the qPCR assay. This finding suggests that either, (a) qPCR was not able to detect some lowdensity midgut oocyst infections or, (b) as noted by others ${ }^{33}$, there is potential for oocyst misidentification by microscopy in a field laboratory setting. Altogether, these results indicate that Microsporidia $M B$ reduces the establishment of Plasmodium oocysts in the Anopheles midguts and impairs the colonization of Anopheles salivary glands by Plasmodium sporozoites.

Microsporidia MB does not confer a fitness cost. The fecundity and egg to adult survival rate of Microsporidia $M B$ infected and uninfected iso-female lineages were examined. No significant differences were observed in the number of eggs laid by Microsporidia $M B$ infected versus uninfected individuals, indicating that unlike most other mosquito microsporidians ${ }^{17}$, Microsporidia $M B$ does not have a sterilizing effect on females (Fig. 5a). A shortened development period from egg to adult was observed for individuals carrying Microsporidia $M B$ (Fig. 5b, c). We investigated the survival of adult female mosquitoes harboring Microsporidia $M B$ and found their longevity was similar to uninfected mosquitoes (Fig. 5d). The intensity of Microsporidia $M B$ was examined across the lifecycle of the mosquito and was higher in adults than in larvae, but larval intensity is highly variable. Notably, Microsporidia $M B$ levels are lower in recently emerged adults, increasing with mosquito age (Fig. 5e).

Microsporidia MB effects gene expression in An. arabiensis. To gain insights into the mechanistic basis of the Microsporidia $M B$ Plasmodium protection, RNA sequencing was carried out in samples of pooled An. arabiensis midguts harboring the Microsporidia $M B$ symbiont and uninfected controls (Supplementary Table 2). In addition, RNA sequencing was carried out in pooled 


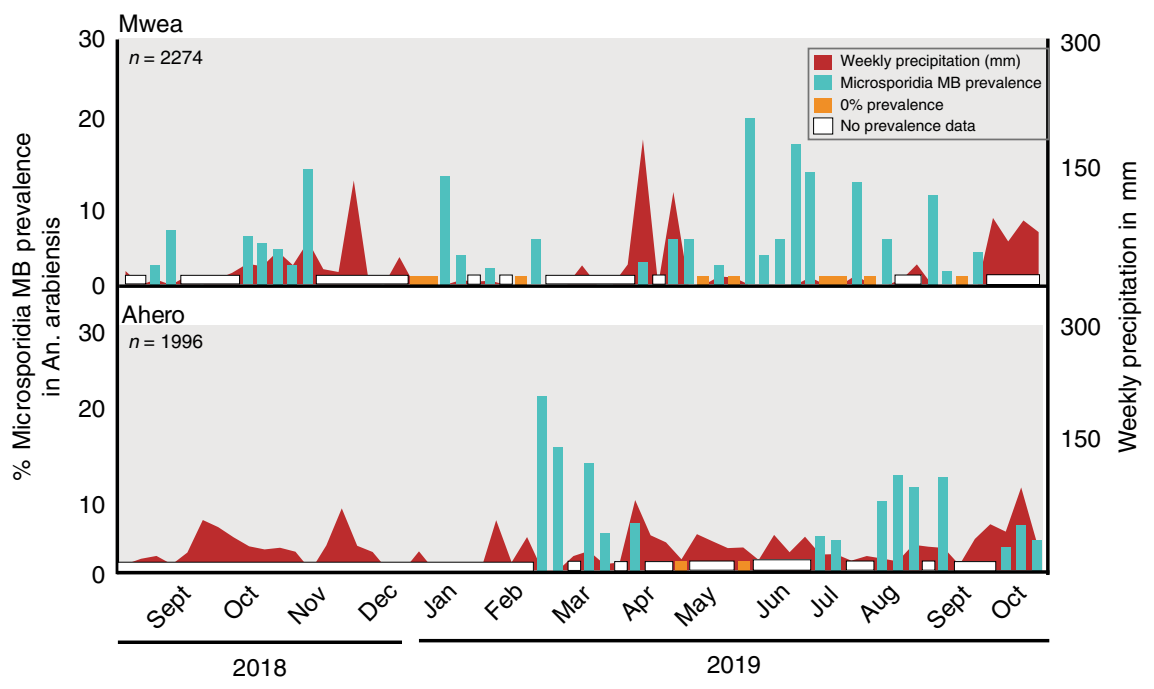

Fig. 2 Seasonal variation in Microsporidia MB prevalence in two sampling sites. Weekly precipitation values given are based on earth observation and are shown in dark blue. Light blue bars indicate the prevalence of Microsporidia MB, monitored in weekly collections. Overall, 136 out of 2274 (6\%) female An. arabiensis were found to be infected with Microsporidia MB in Mwea and 159 out of 1996 (8\%) female An. arabiensis in Ahero. Source data are provided as a Source Data file.

samples of An. arabiensis ovaries, since these tissues were found to have a high intensity of Microsporidia $M B$ infection (Supplementary Table 3). Full-length RNA sequencing (Oxford Nanopore Technologies) was used to identify candidate genes involved in impairing Plasmodium transmission. Out of 13,166 transcripts from the An. arabiensis (AARA) transcriptome, 8611 transcripts were detected with $\geq 10$ reads. We found that genes transcribed at higher levels in $A n$. arabiensis midguts in the presence of Microsporidia $M B$ compared to uninfected midguts were predominately involved in digestion and immunity. In the midgut the most upregulated genes in the presence of Microsporidia $M B$ were the serine proteases ISP13 (AARA001672-RA), antryp-4 (AARA016538-RA) and antryp-1 (AARA016534-RA), the female reproductive tract protease GLEANR_896 (AARA009400-RA), and the anti-microbial peptides cecropin-2 (AARA015679-RA) and gambicin (AARA010954-RA). In the ovaries, Microsporidia $M B$ was associated with high levels of c-type lysozyme (AARA016504-RA), transferrin (AARA010742-RA) and Salivary gland (AARA001017-RA, AARA008387-RA) and Salivary glandlike protein (AARA016177-RA) genes.

\section{Discussion}

Several Anopheles-associated microsporidians have been shown to interfere with the infection and development of Plasmodium ${ }^{18,34,35}$. Nosema stegomyiae disrupts the development of the oocysts in An. gambiae, attributed to midgut degradation and consequent disruption of Plasmodium binding ${ }^{18}$, while Vavraia culicis impairment of development of Plasmodium has been associated with host innate immune priming ${ }^{35}$. The impairment observed in previous Plasmodium transmission experiments have been relatively modest, with only partial reduction of transmission observed. In applied terms, given their virulence, their primary application would be as population suppression agents and the Plasmodium transmission impairment would provide a minor add-on. In contrast, high levels of impairment of $P$. falciparum transmission were observed here for Microsporidia MB. Additionally, Microsporidia $M B$ interferes with Plasmodium development early, prior to the formation of Plasmodium oocysts in the Anopheline mosquito gut.

Microsporidia $M B$ infected female An. arabiensis transmit the infection with high efficiency to their offspring. Microsporidia $M B$ were observed in $A n$. arabiensis ovaries suggesting a transovarial transmission route. It is notable that a number of females with low Microsporidia $M B$ intensity did not transmit or transmitted very poorly to their offspring. It is possible that these infections might be newly horizontally acquired and have not yet become localized to the ovaries, a likely requirement for high-efficiency maternal transmission. The identification of Microsporidia MB spores in the midgut is suggestive of a horizontal transmission route, which is most likely to take place in larval habitats or potentially during mosquito mating. Environmental conditions appear to influence Microsporidia $M B$ infection prevalence in the field, because the highest infection rates were generally observed 4-6 weeks after peak rainfall. It is conceivable that the rates of vertical, and possibly horizontal, transmission are influenced by environmental factors.

Microsporidia $M B$ infection does not have an overt effect on An. arabiensis fitness, as females harboring Microsporidia $M B$ laid an equivalent number of eggs and had a similar lifespan to uninfected controls. Vertically transmitted symbionts are generally selected to minimize fitness impacts on their hosts ${ }^{36}$ and this could explain Microsporidia $M B$ levels being lower in larvae and increasing modestly across the life of adult An. arabiensis. The fitness of adult mosquitoes is highly correlated to their size at eclosion $^{37}$ and maintaining low Microsporidia MB infection levels in larvae could be important to lessen potential impacts on larval growth rate and hence minimize overall host fitness cost. An. arabiensis infected with Microsporidia $M B$ were found to have a slightly faster larval development period. It is notable that another microsporidian Vavraia culicis, also shortened mosquito development time in Culex pipiens but in contrast to Microsporidia $M B$ this was associated with a high fitness $\operatorname{cost}^{38}$. Microsporidia $M B$ could also be affecting host metabolic processes or nutrient availability leading to more rapid host growth, as has been suggested for other vertically transmitted symbionts that have mutualistic phenotypes in An. gambiae ${ }^{39}$.

Elucidating the mechanistic basis of endosymbiont-induced protective phenotypes presents a number of challenges. In systems where these mechanisms have been investigated, there are indications that multiple processes may be involved ${ }^{40}$. To gain insight into the Plasmodium-protective phenotype associated with Microsporidia $M B$, we investigated changes in host gene 
a
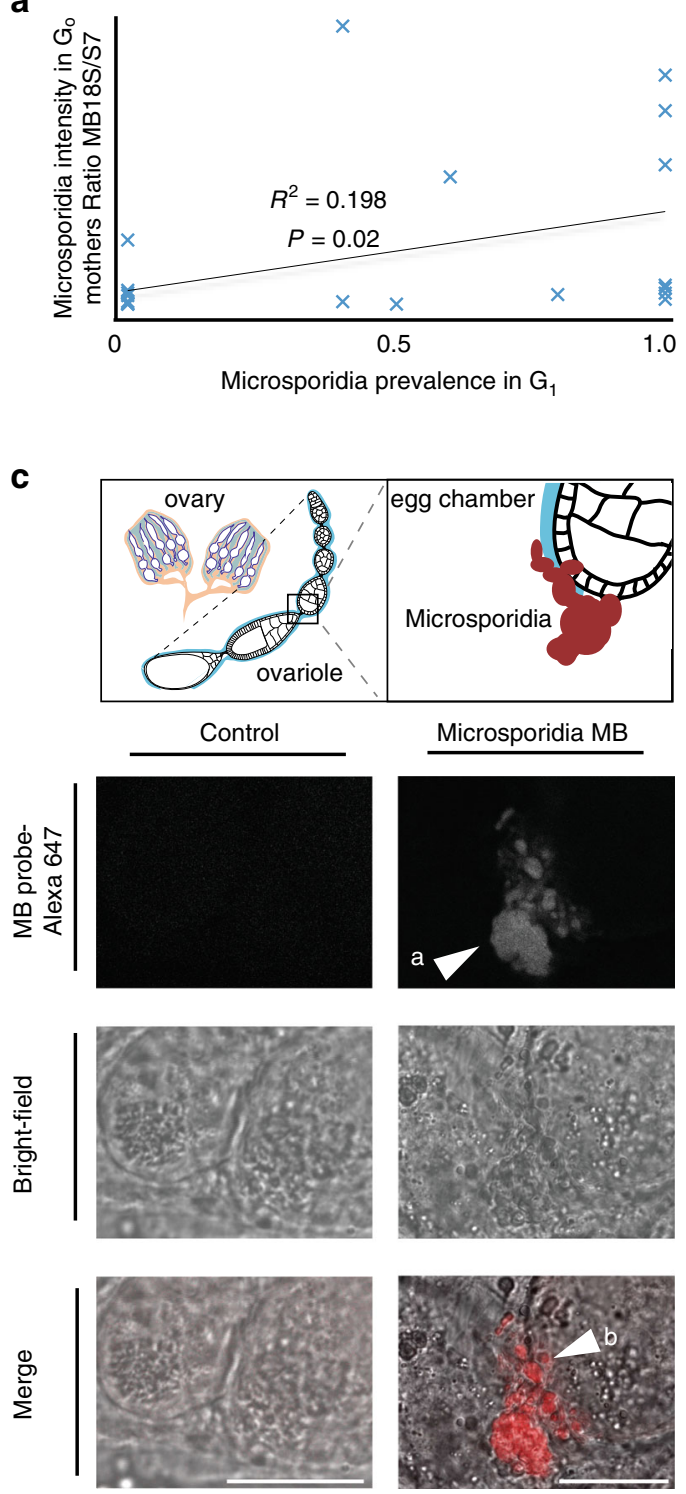

b

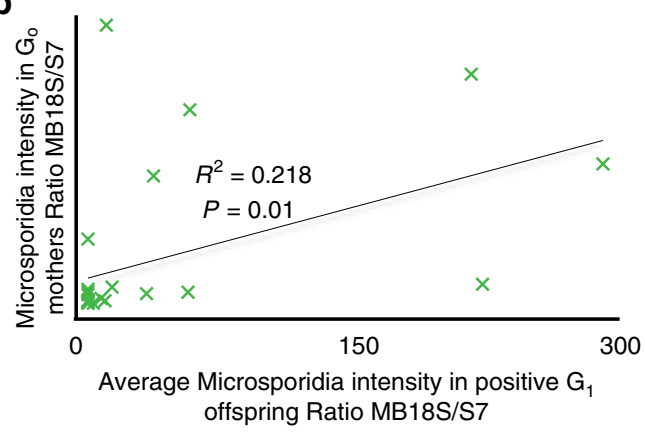

d

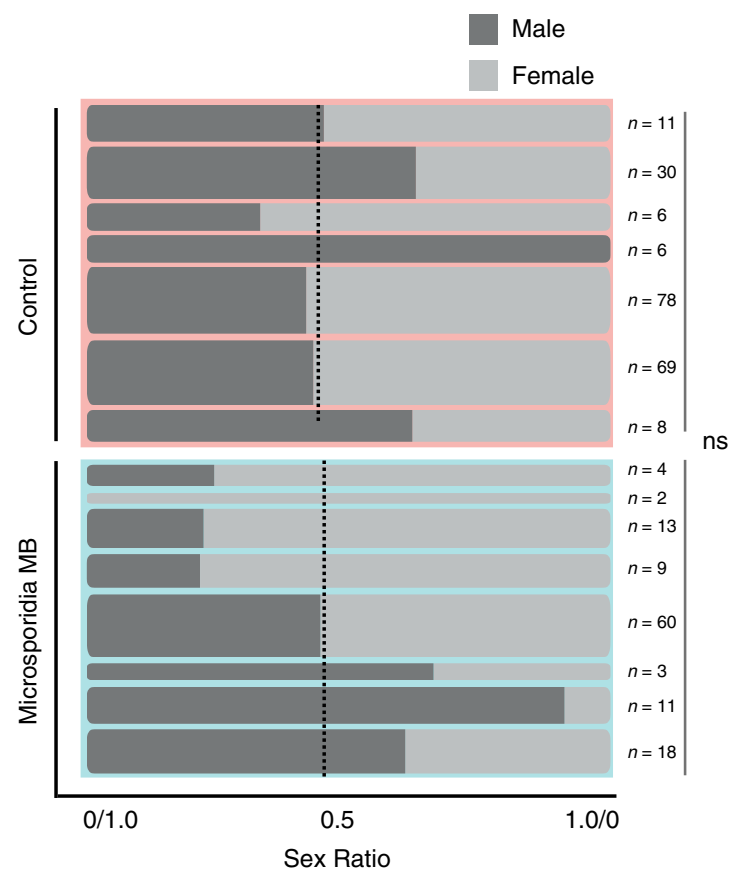

Fig. 3 Microsporidia MB is maternally transmitted and does not bias sex ratio. a The vertical transmission efficiency of Microsporidia $M B$ to $G_{1}$ depends on the $G_{0}$ (maternal) Microsporidia $M B$ intensity. In a linear regression, the slope is significantly non-zero $(P=0.02, F=6.169$, and $n=27)$. $\mathbf{b}$ The intensity of Microsporidia $M B$ in $G_{1}$ offspring is correlated to $G_{0}$ maternal intensity, with a linear regression the slope that is significantly non-zero $(P=0.01, F=6.98$ and $n=27$ ). c Fluorescence microscopy with a Microsporidia MB specific FISH probe (MB probe) indicates that Microsporidia MB (a) is localized to the posterior of developing vitellogenic egg chambers (b) in An. arabiensis. Scale bar, $20 \mu \mathrm{m}$. Representative images as observed in three independent experiments. d The sex ratio of broods from Microsporidia $M B$ infected mothers does not differ significantly from Microsporidia MB uninfected mothers suggesting there is no sex ratio distortion (Unpaired two-tailed $t$-test, $P=0.32, t=1.02, \mathrm{df}=13$ ). Dashed line reflects overall sex ratio (Uninfected control $=100: 108$ or $52 \%$ female, Microsporidia $M B=56: 64$ or $54 \%$ female). Column widths reflect sample sizes Source data are provided as a Source Data file.

expression. Microsporidia $M B$ apparently increased the expression of a number of genes, which are involved in digestion (antryp-4, antryp-1) and immunity (ISP13, cecropin-2 and gambicin $)^{41,42}$. The genes ISP13 and cecropin-2 have been shown to be upregulated in response to infection ${ }^{40,43}$ and gambicin has been shown to be an anti-Plasmodium factor in An. gambiae ${ }^{44}$, which lends support to the hypothesis that the impairment of Plasmodium transmission by Microsporidia $M B$ could be linked to immune priming. Antryp-4 is a digestive serine protease that is constitutively expressed in unfed $A n$. gambiae mosquitoes, whereas antryp-1 is upregulated upon blood-feeding 41 . The finding that antryp-4 and antryp-1 are upregulated in the presence of Microsporidia $M B$ suggests that this microsporidian could affect host metabolism, which may also have implications for vector capacity ${ }^{45-47}$.

These findings are significant in terms of regional malaria transmission and epidemiology as well as risk-mapping, and particularly in terms of the development of microbe-based Plasmodium transmission-blocking tools. Microsporidia $M B$ can be added to the Anopheles-associated gut bacteria that have previously been identified as able to reduce Plasmodium transmission when introduced into the mosquito ${ }^{48,49}$, or following introduction of anti-Plasmodium transgenes ${ }^{50}$. In addition, the impairment of Plasmodium transmission combined with the key characteristics that will facilitate artificially elevating its population frequency, namely spore production that is likely to facilitate 
a

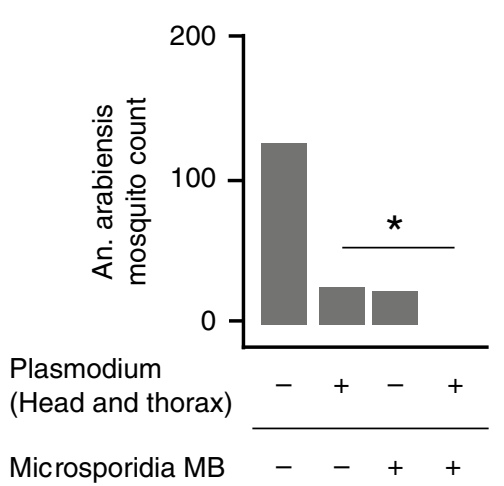

C

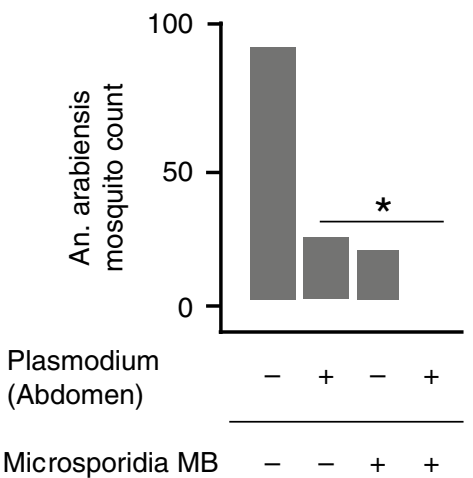

b

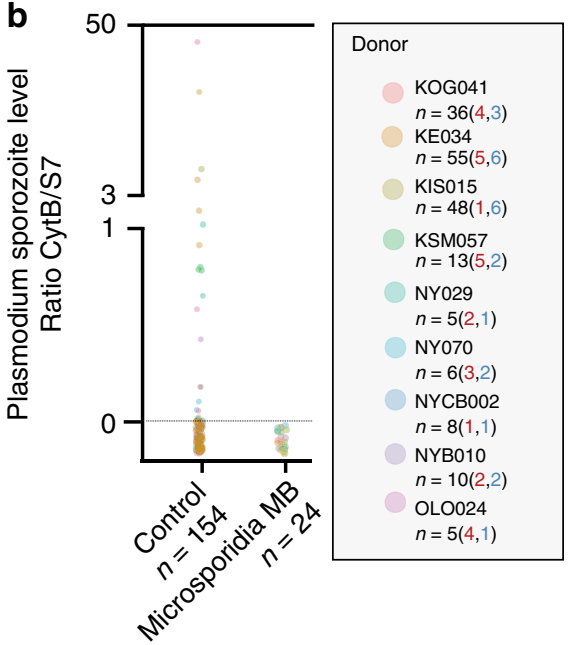

d

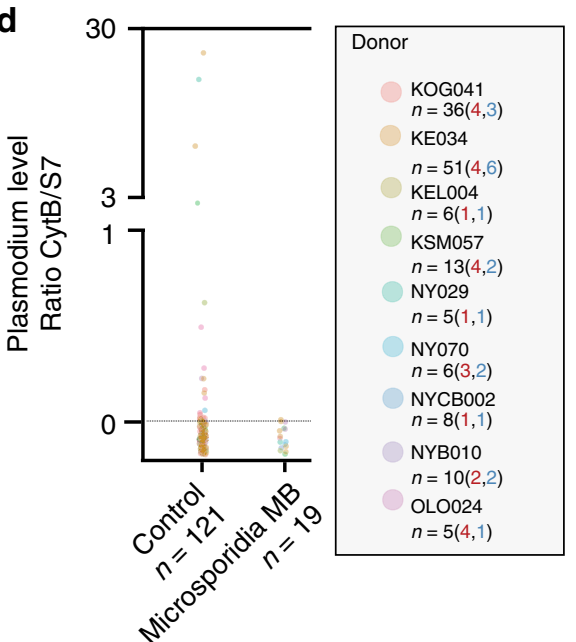

Fig. 4 Microsporidia MB impairs parasite development in An. arabiensis after direct membrane feeding assay challenge with $\boldsymbol{P}$. falciparum. The Plasmodium infection rate in Microsporidia MB positive and Microsporidia MB negative mosquitoes was determined by $\mathrm{qPCR}$. a The head and thorax Plasmodium infection rate, reflecting presence of sporozoites, in Microsporidia MB positive and Microsporidia MB negative mosquitoes. There was a significant absence of co-infected mosquitoes (two-tailed fisher exact test, $P=0.02 n=178$ ). $\mathbf{b}$ Plasmodium intensity in An. arabiensis heads and thoraxes, quantified by GPCR. $\mathbf{c}$ The abdomen Plasmodium infection rate, reflecting presence of oocysts, in Microsporidia MB positive and Microsporidia MB negative mosquitoes. There was a significant absence of co-infected mosquitoes (two-tailed fisher exact test, $P=0.04, n=140$ ). $\mathbf{d}$ Plasmodium intensity in $A n$. arabiensis abdomens, quantified by qPCR. Data shown in $(\mathbf{a}, \mathbf{b}, \mathbf{c}, \mathbf{d})$ are pooled from replicate experiments carried out using different gametocyte donors (for each donor the numbers of Plasmodium positive An. arabiensis is shown in red and Microsporidia MB positives An. arabiensis is shown in blue). Each data point is an individual An. arabiensis mosquito. Source data are provided as a Source Data file.

dissemination, efficient transovarial transmission and apparently non-virulent interactions with $A n$. arabiensis mosquitoes make Microsporidia $M B$ is a realistic candidate for a stable vector population replacement strategy. As an unmodified, Anophelesassociated inherited endosymbiont, it provides attractive prospects for malaria control.

\section{Methods}

Sampling sites and collection. Microsporidia $M B$ and Plasmodium prevalence in wild An. arabiensis mosquitoes was determined by collecting adult female mosquitoes from sites around Kenya: Mbita (Nyawiya, Mageta and Kirindo), Mwea (Mbui-Njeru), Busia (Funyala) and Ahero (Kigoche). An. arabiensis were collected inside houses and sheds using CDC light traps and by manual aspiration. For the establishment of Microsporidia $M B$ harboring lines gravid mosquitoes were collected solely by manual aspiration inside houses and sheds. All mosquitoes were transported from the field to the icipe-Thomas Odhiambo Campus (iTOC) laboratories and insectaries alive in cages, each sample represents an individual wild caught mosquito.
Mosquito species identification. All experiments were carried out on wild collected Anopheles gambiae sl., which were identified morphologically. In all of the collection sites, An. arabiensis is the most common member of the An. gambiae species complex, with $>97 \%$ of complex members being identified as An. arabiensis The species designation was confirmed using a molecular assay that differentiates An. gambiae s.s. and An. arabiensis using the SINE S200 X6.1 locus ${ }^{51}$. Anopheles samples that were not confirmed to be An. arabiensis were excluded from analysis.

Seasonal fluctuations and climate data. An. arabiensis were collected by aspiration in houses on a weekly basis. Specimens were transported to laboratories in Nairobi and Mbita for species identification and Microsporidia MB screening. Rainfall data for Mwea $(-37.3538 \mathrm{~W},-0.6577 \mathrm{~N})$ and Ahero $(-34.9190 \mathrm{~W}$,

$-0.1661 \mathrm{~N}$ ) were obtained from Climate Engine ${ }^{52}$, Desert Research Institute and University of Idaho, accessed on (01/12/2019), http://climateengine.org.

Determination of molecular phylogeny of Microsporidia MB. Microsporidia MB positive An. arabiensis were initially identified by sequencing $18 S$ amplicons amplified by the SSU rRNA primer pair F: 5'-CACCAGGTTGATTCTGCC-3'; R: $5^{\prime}$-TTATGATCCTGCTAATGGTTC- $3^{\prime}$, which targets phylogenetically diverse microsporidians ${ }^{53}$. The primer pair RPOBMBF $5^{\prime}$-ACAGTAGGTCACTTGAT 
a

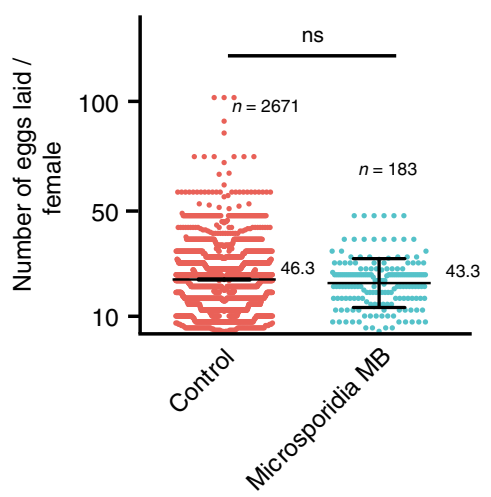

b

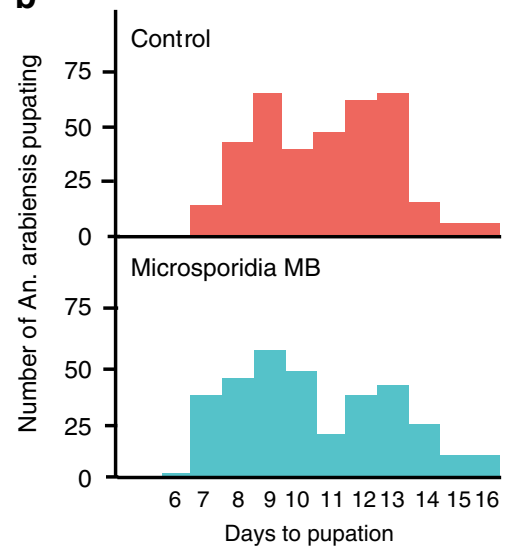

C

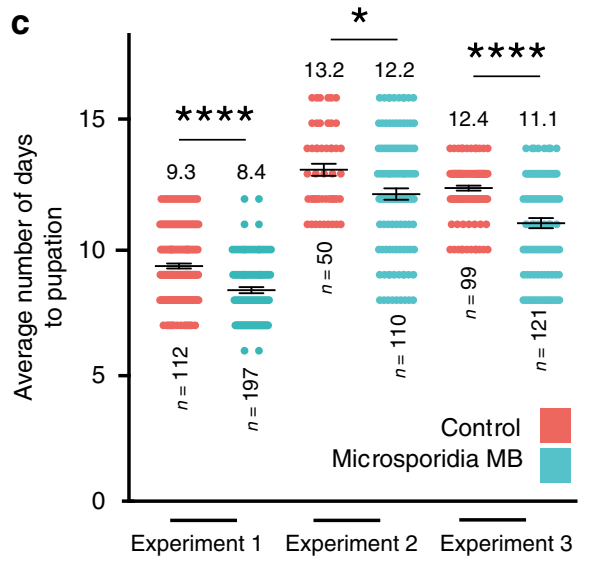

d

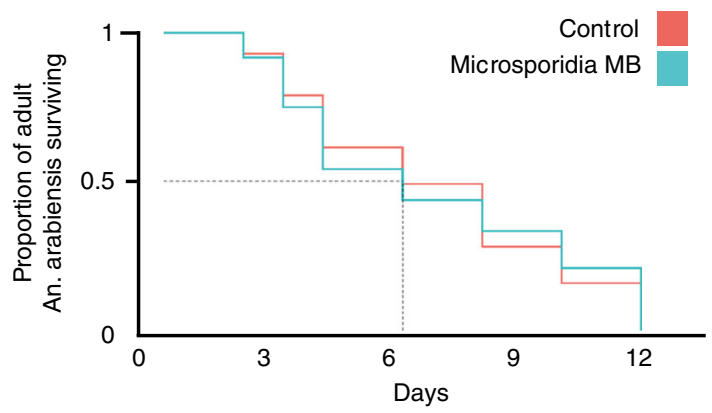

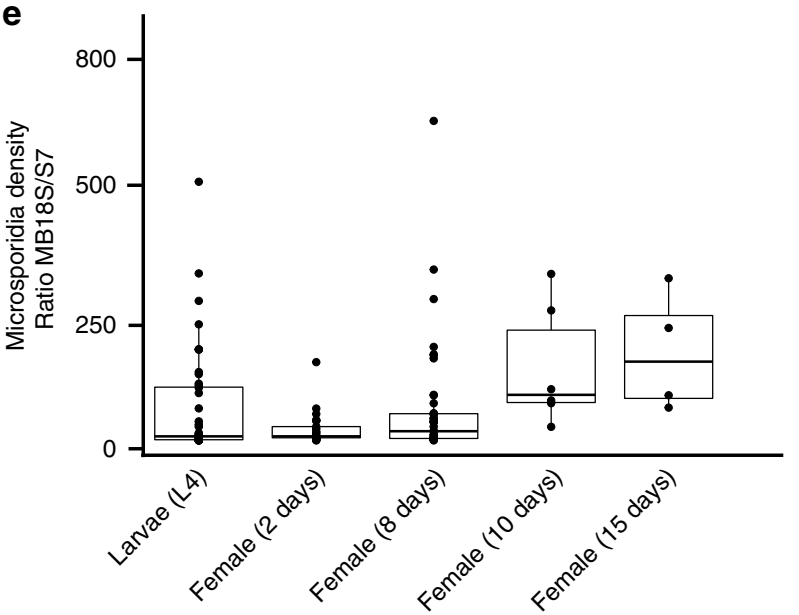

Fig. 5 Microsporidia MB does not overtly decrease host fitness. a Microsporidia MB harboring wild-caught An. arabiensis did not lay significantly less eggs than uninfected counterparts (two-tailed Mann-Whitney test, $P=0.18$ ). The black line indicates the mean and error bars reflect $S E M$. $\mathbf{b}$ The $G_{1}$ larval progeny of Microsporidia MB infected wild-caught females develop significantly faster than uninfected counterparts. c The larval development of Microsporidia $M B$ infected An. arabiensis is on average 1.1, 1.4, and 1.3 days less than uninfected controls in three independent experiments (Two-tailed Mann-Whitney test, $P<0.0001, P=0.03, P<0.0001$, error bars reflect $S E M)$. Mean values are shown above the scatter dot plots. Data shown in (b) and (c) are from the same three independent experiments. $\mathbf{d}$ The survival of adult $\mathrm{G}_{1}$ progeny of Microsporidia MB infected wild-caught females is not significantly different from uninfected counterparts, one experiment (ii) shown of three independent experiments (i-iii); $(\mathrm{i}, P=0.86, n>9$, ii, $P=0.97, n>$ $41, \mathrm{ii}, P=0.06 n>50, n$ denotes the minimum number of mosquitoes per condition). e The intensity of Microsporidia MB across different developmental stages. The intensity of Microsporidia MB is highly variable in larvae, lower in young adults but increases as adult An. arabiensis females age. Boxplot boundaries reflect the inter-quartile range. The horizontal bar is the median and whiskers extend to 1.5 times the inter-quartile range beyond the boxplot. Each datapoint represents an individual mosquito from a total of five (L4-day 2), four (day 8) and three (day 10 and 15) independent experiments. Source data are provided as a Source Data file.

TGAATGTC- ${ }^{\prime}$ and RPOBMBR $5^{\prime}$-TACCATGTGCTTAAGTCTTTGGT-3' was used to amplify the $r p o B$ gene of Microsporidia MB. Amplicons were prepared from nine individual $A n$. arabiensis from geographically dispersed sites, all individuals had identical $18 S$ and $r p o B$ fragment gene sequences. Prepared amplicons were cleaned using the USB ${ }^{\oplus}$ ExoSAP-IT ${ }^{\oplus}$ PCR Product Cleanup kit according to manufacturer's instructions and sent to Macrogen (Netherlands) for sequencing. Multiple sequence alignment was done using the MUSCLE algorithm ${ }^{54}$ version 3.5 in Geneious Prime ${ }^{55}$ version 2020.03 (www.geneious.com) alongside reference sequences of other Microsporidia species obtained from NCBI. Tamura-Nei genetic distance model alongside Neighbour-joining tree building algorithm was used in the creation of phylogenies and evaluated with 10000 replicates bootstrap support and 50\% support threshold. Rooting was done using Conidiobolus osmodes as an outgroup. Microsporidia $M B 18 \mathrm{~S}$ rDNA and rpoB partial gene reference sequences have been submitted to Genbank, submission ID 2308213. The Accession numbers of sequences used for generating phylogenetic trees are given in Supplementary Table 4.

Egg laying and establishment of lines. Wild-caught gravid female mosquitoes were induced to oviposit inside a perforated $1.5 \mathrm{ml}$ micro centrifuge tube containing
$50 \mu \mathrm{l}$ of distilled water and a soaked piece of Whatman paper towel $(1 \mathrm{~cm} \times 1 \mathrm{~cm}$ in size). Eggs from each female were counted under a compound microscope using a paint brush and then dispensed into water tubs for larval development under optimal rearing conditions (a temperature of $30.5^{\circ} \mathrm{C}$ and $30 \%$ humidity). Upon laying eggs, the $\mathrm{G}_{0}$ females were screened for presence of Microsporidia $M B$ by PCR. The progeny from Microsporidia $M B$ positive $\mathrm{G}_{0}$ females were used for: (i) the quantification of vertical transmission efficiency and (ii) the establishment of iso-female Microsporidia $M B$ infected lines, whereas lines with similar L1 larvae numbers ( \pm 10 individuals) were used as matched negative controls for quantifying development time and survival. For each iso-female line two $G_{1} L 4$ larvae were screened to determine species and confirm infection status and the line was only considered infected if both were Microsporidia $M B$ positive. For the quantification of intensity across developmental stages infected iso-female lines were established. We also scored the sex ratio of progeny by allowing specimens to eclose and then counting males and females. To establish mixed (Microsporidia MB positive and negative) An. arabiensis pools for direct membrane feeding assays, eggs from Microsporidia $M B$ infected and uninfected females were combined and reared together at an approximate ratio of 3:1 (Microsporidia $M B$ infected: Microsporidia MB uninfected). Infection status was not determined at the larval stage for mixed pools. 
Mosquito rearing. Larvae were reared under a controlled environment at $30.5^{\circ} \mathrm{C}$ $\left( \pm 2{ }^{\circ} \mathrm{C}\right)$. Larvae were fed daily on TetraMin ${ }^{\mathrm{Tu}}$ baby fish food and fresh doubledistilled water added into their tubs every other day to maintain oxygen levels. Adult mosquitoes were reared at $30^{\circ} \mathrm{C}\left( \pm 2{ }^{\circ} \mathrm{C}\right)$ and $70 \%$ humidity with a constant 12 -h day/night cycle. The adult mosquitoes were fed on $6 \%$ glucose soaked in cotton wool. For adult survival and larval development time measurements, the status of larvae and mosquitoes was recorded every $24 \mathrm{~h}$.

Plasmodium direct membrane feeding assays. Plasmodium screening of human subjects was done in the regions surrounding Mbita using Rapid Diagnostic Test (RDT) kits (SD Bioline, UK). Microscopy was carried out on RDT-positive samples to confirm the presence of P. falciparum gametocytes. Gametocyte-positive blood used was mixed with an anticoagulant (heparin) and a total volume of $500 \mu \mathrm{l}$ was placed into mosquito mini-feeders at $37^{\circ} \mathrm{C}$ and covered in stretched parafilm. Mixed pools of 2-3-day-old An. arabiensis (containing co-reared Microsporidia $M B$ positive and negative mosquitoes) were starved for $5 \mathrm{~h}$ (the sucrose source was replaced with water for the first $4 \mathrm{~h}$ of starvation) prior to the direct membrane feeding assay. Mosquitoes were allowed to feed for an hour after which non bloodfed individuals were discarded. Blood-fed mosquitoes were then maintained for a period of 10 days post-infection and processed for the molecular detection of Microsporidia $M B$ and Plasmodium oocysts and sporozoites. We only include experiments where there was greater than zero prevalence of Microsporidia $M B$ and Plasmodium in the mixed pools of An. arabiensis.

Specimen storage and DNA extraction. All An. arabiensis specimens were dry frozen at $-20^{\circ} \mathrm{C}$ in individual microcentrifuge tubes prior to DNA extraction. Prior to extraction, mosquitoes were sectioned into head and thorax (for detection and quantification of Plasmodium sporozoites and Microsporidia MB) and abdomens (for detection and quantification of Plasmodium oocysts and Microsporidia $M B)$. DNA was extracted from each section individually using the protein precipitation method (Puregene, Qiagen, Netherlands).

Molecular detection and quantification of Microsporidia MB. Microsporidia MB specific primers (MB18SF: CGCCGGCCGTGAAAAATTTA and MB18SR: CCTTGGACGTGGGAGCTATC) were designed to target the Microsporidia $M B$ $18 S$ rRNA gene region and tested for specificity on a variety of Microsporidiainfected mosquito controls (including Hazardia, Parathelohania and Takaokaspora). For detection, the PCR reaction volume was $10 \mu \mathrm{l}$, consisting of $2 \mu \mathrm{l}$ HOTFirepol $^{\circledast}$ Blend Master mix Ready-To-Load (Solis Biodyne, Estonia, mix composition: $7.5 \mathrm{mM}$ Magnesium chloride, $2 \mathrm{mM}$ of each dNTPs, HOT FIREPol ${ }^{\varpi}$ DNA polymerase), $0.5 \mu \mathrm{l}$ of $5 \mathrm{pmol} \mu^{-1}$ of both forward and reverse primers, $2 \mu \mathrm{l}$ of the template and $5 \mu \mathrm{l}$ nuclease-free PCR water. The PCR cyclic conditions used were; initial denaturation at $95^{\circ} \mathrm{C}$ for $15 \mathrm{~min}$, further denaturation at $95^{\circ} \mathrm{C}$ for $1 \mathrm{~min}$, followed by annealing at $62^{\circ} \mathrm{C}$ for $90 \mathrm{~s}$ and extension at $72^{\circ} \mathrm{C}$ for a further $60 \mathrm{~s}$, all done for 35 cycles. Final elongation was done at $72{ }^{\circ} \mathrm{C}$ for $5 \mathrm{~min}$. Microsporidia $M B$ was also quantified by qPCR using MB18SF/ MB18SR primers, with normalization against the Anopheles ribosomal $S 7$ host gene ${ }^{56}$ (primers, S7F: TCCTGGAGCTGGAGATGAAC and S7R: GACGGGTCTGTACCTTCTGG). The primer efficiencies were determined using published methods ${ }^{57}$. Samples were considered negative if the cycle threshold $(\mathrm{Ct})$ value was greater than 35 or if the melt curve did not align with the positive control. These conditions were met for all negative control samples, which was DNA extracted from An. arabiensis from the insectary at icipe, Nairobi. The qPCR reaction volume was $10 \mu \mathrm{l}$, consisting of $2 \mu \mathrm{l}$ HOT FIREPol ${ }^{\circledast}$ EvaGreen $^{\oplus}$ HRM no ROX Mix (Solis Biodyne, Estonia, mix composition: $12.5 \mathrm{mM}$ Magnesium chloride, EvaGreen ${ }^{\oplus}$ dye, BSA, dNTPs, HOT FIREPol $^{\oplus}$ DNA Polymerase and $5 \times$ EvaGreen $^{\circledast}$ HRM buffer), $0.5 \mu \mathrm{l}$ of $5 \mathrm{pmol}^{-1}$ of both forward and reverse primers, $2 \mu \mathrm{l}$ of the template and $5 \mu \mathrm{l}$ nuclease-free PCR water. Finally, melt curves were generated including temperature ranges from $65^{\circ} \mathrm{C}$ to $95^{\circ} \mathrm{C}$. Standard curves were also generated to determine amplification efficiency. An. arabiensis were considered to be infected if Microsporidia MB was detected in either head and thorax or abdomen compartments. All reactions were carried out on a MIC qPCR cycler (Bio Molecular Systems, Australia).

\section{Molecular detection and quantification of Plasmodium. A qPCR-based assay} was used to detect and quantify the cytochrome $b$ gene of Plasmodium ${ }^{58}$. When used on An. arabiensis head and thorax DNA samples 10 days post DMFA this assay can be used to detect and quantify Plasmodium sporozoites. When used on An. arabiensis abdomen DNA samples 10 days post DMFA this assay can be used to detect and quantify Plasmodium oocysts ${ }^{30}$. To confirm that the qPCR-based assay was able to detect oocysts in a manner comparable to microscopy, midguts were dissected, stained and oocysts counted prior to them being removed from slides for DNA extraction and $\mathrm{qPCR}^{59}$ (Supplementary Fig. 4). Plasmodium cytochrome $b$ was normalized against the Anopheles ribosomal S7 host gene ${ }^{56}$ Samples were considered negative if the cycle threshold $(\mathrm{Ct})$ value was greater than 35 or if the melt curve did not align with the positive control. These conditions that were met for all negative control samples, which was DNA extracted from An. arabiensis from the insectary at icipe iTOC, Mbita, fed on heat-inactivated Gametocyte-positive blood 10 days prior to extraction. The qPCR mastermix was composed of $2 \mu$ l HOT FIREPol $^{\oplus}$ EvaGreen $^{\oplus}$ HRM no ROX Mix (Solis Biodyne,
Estonia, mix composition: $12.5 \mathrm{mM}$ Magnesium chloride, EvaGreen dye, BSA, dNTPs, HOT FIREPol ${ }^{\oplus}$ DNA Polymerase and $5 \times$ EvaGreen $^{\oplus}$ HRM buffer), $0.5 \mu$ l of $5 \mathrm{pmol}^{-1}$ of both forward (cytBF) and reverse (cytBR) primers, $2 \mu \mathrm{l}$ of the template and $5 \mu \mathrm{l}$ nuclease-free PCR water. The PCR profile for target gene amplification included an initial denaturation at $95^{\circ} \mathrm{C}$ for $15 \mathrm{~min}$, further denaturation at $95^{\circ} \mathrm{C}$ for $30 \mathrm{~s}$, followed by annealing at $60^{\circ} \mathrm{C}$ for $45 \mathrm{~s}$ and extension at $72{ }^{\circ} \mathrm{C}$ for $45 \mathrm{~s}$ repeated for 40 cycles. Final elongation was performed at $72{ }^{\circ} \mathrm{C}$ for 7 min followed by generation of a melt curve ranging from $65^{\circ} \mathrm{C}$ to $95^{\circ} \mathrm{C}$. A standard curve was generated to determine the PCR efficiency. All reactions were carried out on a MIC qPCR cycler (Bio Molecular Systems, Australia).

Fluorescence in situ hybridization (FISH). DNA probes specific to Microsporidia $M B 18 S$ rDNA were designed and synthesized (5'-CY5-CCCTGTCCACTATACCTAATGAACAT- $3^{\prime}$, Macrogen, Netherlands). FISH was conducted on An. arabiensis adult and larval tissue specimens using a previously described proto$\mathrm{col}^{60}$, with minor modifications. Briefly, mosquito tissues (larval gut and adult ovaries) were fixed in $4 \%$ Paraformaldehyde (PFA) solution overnight at $4{ }^{\circ} \mathrm{C}$ and subsequently transferred into $10 \%$ hydrogen peroxide in $6 \%$ alcohol for 3 days prior to rehydration in Phosphate Buffer Saline with Tween-20 (PBS-T) for 1-2 h Hybridization was conducted by incubating tissues in $150 \mu$ hybridization buffer (20 mM Tris-HCl [pH 8.0], 0.9 M NaCl, $0.01 \%$ sodium dodecyl sulfate, $30 \%$ formamide) containing $100 \mathrm{pmol} \mathrm{ml}^{-1}$ of the probe at room temperature overnight. After washing with PBS-T, the hybridized samples were placed on a slide and were visualized immediately using a Leica SP5 confocal microscope (Leica Microsystems, USA). Images were analyzed with the Image $1.50 \mathrm{i}$ software package ${ }^{61}$.

RNA sequencing. Two pools of tissue samples from Microsporidia $M B$ infected An. arabiensis (3 midguts, 12 ovaries) and two pools of non-infected An. arabiensis (5 midguts, 3 ovaries) were used for RNA extraction. TRIzol ${ }^{\mathrm{TN}}$ reagent (Invitrogen) was used to extract both RNA and DNA from the same An. arabiensis pooled midgut samples. RNA was precipitated with isopropanol, then DNA was extracted from remaining organic and interphase by adding TNES back-extraction buffer (BEB) prior to precipitation with isopropanol. After two 75\% ethanol cleans, DNA and RNA was resuspended in nuclease-free water and quantified with nanodrop. DNA and RNA quality were assessed by checking the size distribution on an electrophoresis-based Bioanalyzer instrument and high-sensitivity DNA and RNA 6000 Nano kits, respectively. RNA was used for long-read RNA sequencing while DNA was used to confirm Microsporidia MB infection by PCR. Extracted RNA was released to the long-read team of the Wellcome Sanger Institute for PCR-cDNA library preparation following manufacturer's instructions (Oxford Nanopore Technologies, UK). $50 \mathrm{ng}$ of total RNA was used for library preparations. Longreads from ONT were then aligned against the An. arabiensis transcriptome (Dongola_AaraD1.11) reference using minimap2 -map-ont. Mapped reads were then used to estimate transcript abundance manually using samtools-1.6 (samtools-1.6 view sorted_bam -f 2 -q $5 \mid$ cut -f $3 \mid$ sort $\mid$ uniq -c $>$./counts/count.txt). The total number of reads obtained was 3,061,001 for the Microsporidia MB infected midgut pool, 195,223 for the uninfected midgut pool, 1,820,596 for the Microsporidia $M B$ infected ovary pool and 390,355 for the uninfected ovary pool. Reads from both pools were randomly resampled to until the total number for each pool was equal to the uninfected read count, which was lower, for each tissue type. The genes corresponding to these reads were then ranked in order of abundance in the Microsporidia $M B$ infected resampled counts relative to absence in Microsporidia $M B$ uninfected resampled counts.

Statistical analysis. We carried out statistical analyses using the two-tailed unpaired t-test to compare unpaired data values with a normal distribution. Where data distributions were non-normal, the two-tailed Mann-Whitney u test was used. Linear regression was used to determine correlation coefficients and significance. To analyze the significance of contingency table data, a two-tailed Fisher exact test was used. The Log-Rank test was used to determine levels of statistical significance for survival data. All statistical analyses were preformed using Graphpad Prizm version $6.0 \mathrm{c}$ software or $\mathrm{R}$ (version 3.5.3). $P$-values of $* p<0.05, * * p<0.01, * * * p<$ 0.001 and $* * * * p<0.0001$ were deemed to be statistically significant.

Ethics statement. Ethical clearance (Kenya Medical Research Institute Scientific and Ethics Review Unit: KEMRI/RES/7/3/1 and Glasgow MLVS College Ethics Committee: Project Number 200170001) was obtained prior to human blood sample collection. Written informed consent was sought from parents and guardians of the children to allow minors to participate in the study. Consent was also obtained from heads of households that provided approval for indoor mosquito collection.

Reporting summary. Further information on research design is available in the Nature Research Reporting Summary linked to this article.

\section{Data availability}

All data supporting the findings of this study are available within the article and its Supplementary Information files, or are available from the authors upon request. The 
source data used to generate Figs. 2-5 and Supplementary Figs. 1-3 and Supplementary Tables 1-3 is available in the Source Data file. The Microsporidia MB $18 \mathrm{~S}$ partial gene sequence has been submitted to Genbank with accession number MT160806[https:// www.ncbi.nlm.nih.gov/nuccore/MT160806]. The Anopheles arabiensis RNA sequencing data have been submitted to the NCBI Sequence Read Archive (SRA) database under accession number PRJNA622655 [https://www.ncbi.nlm.nih.gov/sra/? term $=$ PRJNA622655].

Received: 20 September 2019; Accepted: 11 April 2020;

Published online: 04 May 2020

\section{References}

1. WHO. World Malaria Report (World Health Organization, 2018).

2. Moreira, L. A. et al. A Wolbachia symbiont in Aedes aegypti limits infection with dengue, Chikungunya, and Plasmodium. Cell 139, 1268-1278 (2009).

3. Bian, G., Xu, Y., Lu, P., Xie, Y. \& Xi, Z. The endosymbiotic bacterium Wolbachia induces resistance to dengue virus in Aedes aegypti. PLoS Pathog. 6 , e1000833 (2010).

4. Walker, T. et al. The wMel Wolbachia strain blocks dengue and invades caged Aedes aegypti populations. Nature 476, 450-453 (2011).

5. Hoffmann, A. A. et al. Successful establishment of Wolbachia in Aedes populations to suppress dengue transmission. Nature 476, 454-457 (2011).

6. Frentiu, F. D. et al. Limited dengue virus replication in field-collected Aedes aegypti mosquitoes infected with Wolbachia. PLoS Negl. Trop. Dis. 8, e2688 (2014).

7. Ant, T. H., Herd, C. S., Geoghegan, V., Hoffmann, A. A. \& Sinkins, S. P. The Wolbachia strain wAu provides highly efficient virus transmission blocking in Aedes aegypti. PLoS Pathog. 14, 1-19 (2018).

8. Baldini, F. et al. Evidence of natural Wolbachia infections in field populations of Anopheles gambiae. Nat. Commun. 5, 3985 (2014).

9. Jeffries, C. L. et al. Novel Wolbachia strains in Anopheles malaria vectors from Sub-Saharan Africa. Wellcome Open Res. 3, 113 (2018).

10. Gomes, F. M. et al. Effect of naturally occurring Wolbachia in Anopheles gambiae s.l. mosquitoes from Mali on Plasmodium falciparum malaria transmission. Proc. Natl Acad. Sci. 114, 12566-12571 (2017).

11. Shaw, W. R. et al. Wolbachia infections in natural Anopheles populations affect egg laying and negatively correlate with Plasmodium development. Nat. Commun. 7, 11772 (2016)

12. Gomes, F. M. \& Barillas-Mury, C. Infection of anopheline mosquitoes with Wolbachia: implications for malaria control. PLoS Pathog. 14, e1007333 (2018).

13. Chrostek, E. \& Gerth, M. Is Anopheles gambiae a natural host of Wolbachia? mBio 10, e00784-19 (2018).

14. Han, B. \& Weiss, L. M. Microsporidia: obligate intracellular pathogens within the Fungal Kingdom. Microbiol. Spectr. 5, 2 (2017).

15. Zilio, G., Thiévent, K. \& Koella, J. C. Host genotype and environment affect the trade-off between horizontal and vertical transmission of the parasite Edhazardia aedis. BMC Evol. Biol. 18, 59 (2018).

16. Ironside, J. E. \& Alexander, J. Microsporidian parasites feminise hosts without paramyxean co-infection: support for convergent evolution of parasitic feminisation. Int. J. Parasitol. 45, 427-433 (2015).

17. Becnel, J. J. in Host Regulated Developmental Mechanisms in Vector Arthropods (eds. Borovsky, D. \& Spielman, A.) 240-245 (University of Florida, Gainesville, FL, 1993).

18. Fox, R. M. \& Weiser, J. A Microsporidian Parasite of Anopheles gambiae in Liberia. J. Parasitol. 45, 21 (1959).

19. Andreadis, T. G. \& Hall, D. W. Development, ultrastructure, and mode of transmission of Amblyospora sp. (Microspora) in the Mosquito. J. Protozool. 26, 444-452 (1979).

20. Hulls, R. H. The adverse effects of a microsporidan on sporogony and infectivity of Plasmodium berghei. Trans. R. Soc. Trop. Med. Hyg. 65, 421-422 (1971).

21. Ward, R. A. \& Savage, K. E. Effects of microsporidian parasites upon anopheline mosquitoes and malarial infection. Proc. Helminthol. Soc. Wash. 39, 434-438 (1972).

22. Undeen, A. H. \& Alger, N. E. The effect of the microsporidan, Nosema algerae, on Anopheles stephensi. J. Invertebr. Pathol. 25, 19-24 (1975).

23. Haq, N., Reisen, W. K. \& Aslamkhan, M. The effects of Nosema algerae on the horizontal life table attributes of Anopheles stephensi under laboratory conditions. J. Invertebr. Pathol. 37, 236-242 (1981).

24. Margos, G., Maier, W. A. \& Seitz, H. M. The effect of nosematosis on the development of Plasmodium falciparum in Anopheles stephensi. Parasitol. Res. 78, 168-171 (1992).
25. Schenker, W., Maier, W. A. \& Seitz, H. M. The effects of Nosema algerae on the development of Plasmodium yoelii nigeriensis in Anopheles stephensi. Parasitol. Res. 78, 56-59 (1992).

26. Koella, J. C. \& Agnew, P. Blood-Feeding success of the mosquito Aedes aegypti depends on the transmission route of its parasite Edhazardia aedis. Oikos 78, 311-316 (1997).

27. Tokarev, Y. S., Voronin, V. N., Seliverstova, E. V., Pavlova, O. A. \& Issi, I. V Life cycle, ultrastructure, and molecular phylogeny of Crispospora chironomi g.n. sp.n. (Microsporidia: Terresporidia), a parasite of Chironomus plumosus L. (Diptera: Chironomidae). Parasitol. Res. 107, 1381-1389 (2010).

28. Vossbrinck, C. R. \& Debrunner-Vossbrinck, B. A. Molecular phylogeny of the Microsporidia: ecological, ultrastructural and taxonomic considerations. Folia Parasitol. 52, 131-142 (2005).

29. Baton, L. A. \& Ranford-Cartwright, L. C. Spreading the seeds of millionmurdering death: metamorphoses of malaria in the mosquito. Trends Parasitol. 21, 573-580 (2005).

30. Wang, C. Y. T. et al. Assessing Plasmodium falciparum transmission in mosquito-feeding assays using quantitative PCR. Malar. J. 17, 249 (2018).

31. Arez, A. P. et al. Plasmodium sp.: Optimal protocols for PCR detection of low parasite numbers from mosquito (Anopheles sp.) samples. Exp. Parasitol. 94, 269-272 (2000)

32. Berzosa, P. et al. Comparison of three diagnostic methods (microscopy, RDT, and PCR) for the detection of malaria parasites in representative samples from Equatorial Guinea. Malar. J. 17, 333 (2018).

33. Musiime, A. K. et al. Is that a real oocyst? Insectary establishment and identification of Plasmodium falciparum oocysts in midguts of Anopheles mosquitoes fed on infected human blood in Tororo, Uganda. Malar. J. 18, 287 (2019).

34. Gajanana, A., Tewari, S. C., Reuben, R. \& Rajagopalan, P. K. Partial suppression of malaria parasites in Aedes aegypti and Anopheles stephensi doubly infected with Nosema algerae and Plasmodium. Indian. J. Med. Res. 70 417-423 (1979).

35. Bargielowski, I. \& Koella, J. C. A possible mechanism for the suppression of Plasmodium berghei development in the mosquito Anopheles gambiae by the microsporidian Vavraia culicis. PLoS ONE 4, e4676 (2009).

36. Werren, J.H. \& O'Neill, S.L. in Influential Passengers: Inherited Microorganisms and Arthropod Reproduction (eds. O'Neill, S.L., Hoffmann, A. A., \& Werren, J.H.) 1-41 (Oxford University Press, Oxford, 1997).

37. Takken, W. et al. Larval nutrition differentially affects adult fitness and Plasmodium development in the malaria vectors Anopheles gambiae and Anopheles stephensi. Parasit. Vectors 6, 345 (2013).

38. Agnew, P., Bedhomme, S., Haussy, C. \& Michalakis, Y. Age and size at maturity of the mosquito Culex pipiens infected by the microsporidian parasite Vavraia culicis. Proc. R. Soc. Lond. Ser. B: Biol. Sci. 266, 947-952 (1999).

39. Mitraka, E., Stathopoulos, S., Siden-Kiamos, I., Christophides, G. K. \& Louis, C. Asaia accelerates larval development of Anopheles gambiae. Pathog. Glob. Health 107, 305-311 (2013).

40. Ballinger, M. J. \& Perlman, S. J. The defensive Spiroplasma. Curr. Opin. Insect Sci. 32, 36-41 (2019).

41. Muller, H. M., Catteruccia, F., Vizioli, J., Dellatorre, A. \& Crisanti, A. constitutive and blood meal-induced trypsin genes in Anopheles gambiae. Exp. Parasitol. 81, 371-385 (1995)

42. Dimopoulos, G., Richman, A., Müller, H. M. \& Kafatos, F. C. Molecular immune responses of the mosquito Anopheles gambiae to bacteria and malaria parasites. Proc. Natl Acad. Sci. U. S. A. 94, 11508-11513 (1997).

43. Dana, A. N. et al. Differential gene expression in abdomens of the malaria vector mosquito, Anopheles gambiae, after sugar feeding, blood feeding and Plasmodium berghei infection. BMC Genomics 7, 119 (2006).

44. Vizioli, J. et al. Gambicin: a novel immune responsive antimicrobial peptide from the malaria vector Anopheles gambiae. Proc. Natl Acad. Sci. USA 22, 12630-12635 (2001)

45. Lambrechts, L., Chavatte, J. M., Snounou, G. \& Koella, J. C. Environmental influence on the genetic basis of mosquito resistance to malaria parasites. Proc. R. Soc. B Biol. Sci. 273, 1501-1506 (2006).

46. Luckhart, S. \& Riehle, M. A. The insulin signaling cascade from nematodes to mammals: Insights into innate immunity of Anopheles mosquitoes to malaria parasite infection. Dev. Comp. Immunol. 31, 647-656 (2007).

47. Corby-Harris, V. et al. Activation of akt signaling reduces the prevalence and intensity of malaria parasite infection and lifespan in Anopheles stephensi mosquitoes. PLoS Pathog. 6, e1001003 (2010).

48. Cirimotich, C. M. et al. Natural microbe-mediated refractoriness to Plasmodium infection in Anopheles gambiae. Science 332, 855-858 (2011).

49. Dennison, N. J. et al. Functional genomic analyses of Enterobacter, Anopheles and Plasmodium reciprocal interactions that impact vector competence. Malar. J. 15, 425 (2016).

50. Wang, S. et al. Driving mosquito refractoriness to Plasmodium falciparum with engineered symbiotic bacteria. Science 357, 1399-1402 (2017). 
51. Santolamazza, F., Mancini, E., Simard, F., Qi, Y., Tu, Z. \& della Torre, A. Insertion polymorphisms of SINE200 retrotransposons within speciation islands of Anopheles gambiae molecular forms. Malar. J. 7, 163 (2008).

52. Huntington, J. L. et al. Climate Engine: cloud computing and visualization of climate and remote sensing data for advanced natural resource monitoring and process understanding. Bull. Am. Meteorol. Soc. 98, 2397-2410 (2017).

53. Ghosh, K. \& Weiss, L. M. Molecular diagnostic tests for microsporidia. Interdiscip. Perspect. Infect. Dis. 2009, 926521 (2009).

54. Edgar, R. C. MUSCLE: Multiple sequence alignment with high accuracy and high throughput. Nucleic Acids Res. 32, 1792-1797 (2004).

55. Kearse, M. et al. Geneious Basic: an integrated and extendable desktop software platform for the organization and analysis of sequence data. Bioinformatics 28, 1647-1649 (2012).

56. Dimopoulos, G., Seeley, D., Wolf, A. \& Kafatos, F. C. Malaria infection of the mosquito Anopheles gambiae activates immune-responsive genes during critical transition stages of the parasite life cycle. EMBO J. 17, 6115-6123 (1998).

57. Pfaffl, M. W. A new mathematical model for relative quantification in realtime RT-PCR. Nucleic Acids Res. 29, e45 (2001).

58. $\mathrm{Xu}, \mathrm{W}$. et al. SYBR green real-time PCR-RFLP assay targeting the Plasmodium cytochrome B gene - A highly sensitive molecular tool for malaria parasite detection and species determination. PLoS ONE 10, e0120210 (2015).

59. Graumans, W. et al. Semi-high-throughput detection of Plasmodium falciparum and Plasmodium vivax oocysts in mosquitoes using bead-beating followed by circumsporozoite ELISA and quantitative PCR. Malar. J. 16, 356 (2017).

60. Koga, R., Tsuchida, T. \& Fukatsu, T. Quenching autofluorescence of insect tissues for in situ detection of endosymbionts. Appl. Entomol. Zool. 44, 281-291 (2009).

61. Schneider, C. A., Rasband, W. S. \& Eliceiri, K. W. NIH Image to ImageJ: 25 years of image analysis. Nat. Methods 9, 671-675 (2012).

\section{Acknowledgements}

The authors acknowledge Milcah Gitau of icipe Arthropod Rearing and Containment Unit for mosquito rearing assistance. We thank Ibrahim Kiche, Faith Kyengo, Ulrike Fillinger, Dan Masiga, Jandouwe Villinger and Oscar Mbare for assistance and advice and Quan Lin for assistance releasing RNA sequencing data. This work was supported by the Wellcome Trust [107372, 200274, 202888, 206194], the BBSRC [BB/ R005338/1, sub-grant AV/PP015/1], the Scottish Research Council, the Swiss National Science Foundation [P2ELP3_151932], the R. Geigy Foundation, the UK's Department for International Development (DFID); Swedish International Development Cooperation Agency (Sida); the Swiss Agency for Development and Cooperation (SDC); Federal Democratic Republic of Ethiopia and the Kenyan Government.

\section{Author contributions}

J.K.H conceived and designed the majority of the experiments. E.M, L.M., and E.E.M. performed the majority of experiments. J.W.O, E.E.M., J.J., and E.T. collected mosquitoes, screened them and prepared them for experiments. H.B. and M.V.M. contributed to transmission-blocking and molecular identification experiments, respectively. G.N., E.T., and E.E.M prepared samples for RNA sequencing analysis. M.K.N.L and S.P. conducted the RNA sequencing and analysis. J.K.H., S.P.S., E.M., E.E.M., L.M., J.J., M.V.M., V.A.M., M.K.N.L., S.P., and E.T analyzed the data. J.K.H and S.P.S. wrote the manuscript.

\section{Competing interests}

The authors declare no competing interests.

\section{Additional information}

Supplementary information is available for this paper at https://doi.org/10.1038/s41467020-16121-y.

Correspondence and requests for materials should be addressed to J.K.H.

Peer review information Nature Communications thanks the anonymous reviewers for their contribution to the peer review of this work. Peer reviewer reports are available.

Reprints and permission information is available at http://www.nature.com/reprints

Publisher's note Springer Nature remains neutral with regard to jurisdictional claims in published maps and institutional affiliations.

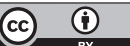

Open Access This article is licensed under a Creative Commons Attribution 4.0 International License, which permits use, sharing, adaptation, distribution and reproduction in any medium or format, as long as you give appropriate credit to the original author(s) and the source, provide a link to the Creative Commons license, and indicate if changes were made. The images or other third party material in this article are included in the article's Creative Commons license, unless indicated otherwise in a credit line to the material. If material is not included in the article's Creative Commons license and your intended use is not permitted by statutory regulation or exceeds the permitted use, you will need to obtain permission directly from the copyright holder. To view a copy of this license, visit http://creativecommons.org/ licenses/by/4.0/.

(C) The Author(s) 2020 